\title{
Trauma and Suicide: The Impact of Direct and Secondary Exposure
}

\author{
Nina Krohne and Meta Lavrič
}

Nina Krohne is a researcher at University of Primorska, Andrej Marusic Institute, Slovene Centre for Suicide Research, where she participates in the implementation of current international and national research and intervention projects in the field of suicidal behaviour and mental health. During the pandemic, she was involved in several research projects, aimed at assessing mental health of various target groups and cooperated in providing psychological support for students in distress and mindfulness courses to general population. She is a PhD candidate and as part of her doctoral dissertation, she studies suicidal behaviour among women with experience of intimate-partner violence. She is a teaching assistant at the Faculty of Mathematics, Natural Sciences and Informational Technology. Meta Lavrič is a young researcher at the Slovene Centre for Suicide Research, where she participates in the implementation of current international and national research and intervention projects in the field of mental health and suicidality. She is also a teaching assistant at the University of Primorska (Faculty of Mathematics, Natural Sciences and Information Technologies) and at the University of Maribor (Faculty of Arts). In her PhD research she focuses mainly on the gatekeepers - their mental health, professional quality of life and a sense of competence in working with suicidal individuals. She is also interested in the use of cognitive-behavioural and mindfulness techniques in mental health interventions for gatekeepers.

Abstract. Various traumatic events have a role in predicting suicidal behaviour. In this presentation we will focus on direct and secondary exposure to traumatic stress, with an emphasis on trauma in interpersonal relationships and trauma related to violent events. More specifically, we will discuss the implications of direct traumatic exposure in intimate-partner violence and of secondary traumatic exposure in gatekeepers working with people who experienced trauma. Exposure to traumatic events can result in long lasting psychological consequences, including post-traumatic stress disorder, depression and anxiety. These psychological outcomes are related to increased risk of suicidal behaviour. The role of mental health problems as a consequence of traumatic experiences in predicting suicide will be discussed in this presentation. We will also discuss the role of trauma in suicidal risk through the 
lens of the interpersonal theory of suicide by Thomas Joiner, specifically the role of trauma in the presence of thwarted belongingness, perceived burdensomeness and acquired capability for suicide. Treating trauma-related suicidality often requires approaches helping a sufferer to overcome the traumatic experiences. Thus, we will raise certain specifics of suicide prevention interventions when working with people who experienced or are experiencing direct or secondary trauma exposure. 